\title{
Air Pollution and Emergency Department Visits for Infectious Diseases in Toronto, Canada
}

\author{
Mieczysław Szyszkowicz ${ }^{1, *}$, Nicholas de Angelis ${ }^{2}$ \\ 1 Environmental Health Science and Research Bureau, Health Canada, Ottawa, Canada \\ 2 Biomedical Program, Department of Mechanical and Aerospace Engineering, Carleton University, Ottawa \\ * Correspondence: mietek.szyszkowicz@canada.ca
}

\begin{abstract}
This study examines the relation between ambient air pollution and emergency department (ED) visits due to certain infectious diseases in Toronto, Canada. The National Ambulatory Care Reporting System database was used to draw the corresponding health cases. Daily data on ED visits, ambient air pollution concentration levels, and weather conditions during the period from April 2004 to December 2015 (4,292 days in total) were linked together and used in statistical models. Six air pollutants (fine particulate matter $\mathrm{PM}_{2.5}, \mathrm{CO}, \mathrm{NO}_{2}, \mathrm{SO}_{2}$, ozone $\mathrm{O}_{3}$ as a daily average, and ozone $\mathrm{O}_{3}-8$ hour ozone, as a maximum eight hour average) were investigated. In addition, the Air Quality Health Index (combining $\mathrm{NO}_{2}, \mathrm{O}_{3}$, and $\mathrm{PM}_{2.5}$ ) was also considered. The time-stratified casecrossover technique was applied in the study design. Conditional Poisson models were created using the daily counts of ED visit data. The considered factors, air pollutants and weather, were lagged by the same number of days, from 0 to 14 . In the period of the study 339,644 ED visits were identified; 177,619 for females and 162,025 for males. For each air pollutant 270 models were realized (15 lags x 18 strata). Ambient air pollution concentrations lagged by 2, 3, and 5 days have the highest impact on ED visits, with 34,32 , and 35 positive associations, respectively. For all patients and an increase in a one interquartile range $(\mathrm{IQR}=1.2 \mathrm{ppb})$ of sulphur dioxide, the following values of the relative risks (RR) were estimated: $R R=1.005$ (95\% confidence interval: 0.998, 1.013), 1.008 (1.001, 1.016), 1.009 (1.001, 1.016), $1.011(1.004,1.019), 1.007(0.987,1.028)$, and $1.009(1.002,1.016)$ for lags from 0 to 5 , respectively. The results suggest that exposures for certain air pollutants (mainly CO, $\mathrm{O}_{3}$, and $\mathrm{SO}_{2}$ ) in urban environment affect the number of ED visits related to infectious diseases.
\end{abstract}

Keywords: ambient air pollution; case-crossover; cluster; concentration; counts; strata; urban

\section{Introduction}

Numerous epidemiologic studies have shown a positive association between elevated concentrations of ambient air pollution and increased risk of health conditions resulting in emergency department (ED) visits, hospital admissions, morbidity, and mortality [1-3]. The majority of these environmental epidemiology studies were focused on pulmonary and cardiac health conditions, and only in recent years, a wider range of diseases have been considered and associated with ambient air pollution exposures [4-6]. Examples include epidemiologic studies supported by the achievements in toxicology, which explain biological plausibility when considering other health conditions, such as metabolic disorders, impaired cognition, dementia, depression, suicide, and many others $[3,7]$. Some correlations between air pollution and health conditions are often unpredictable and unexpected. In practice, after carefully toxicological investigations, the associations are justified [7]. These types of studies are usually conducted as short-term or long-term (cohort studies) exposures to air pollution - the type of study depends on the health conditions and associations being tested. Here, a short-term study is performed with one day as the time unit. 
In this presentation, emergency department (ED) visits for the diseases grouped under a single Chapter ("Certain infectious and parasitic diseases") of the International Classification of Diseases, Tenth Revision (ICD-10) are considered (ICD-10 codes: A00-B99). Specific health problems from this group are not investigated individually but rather all diseases identified within this ICD-10 code group are considered as one and the same health problem $[8,9]$. The main focus of this study is the number of daily ED visits and their correlations with ambient air pollution concentration levels using a large urban agglomeration in Canada - the city of Toronto - as the location of the study.

The tested hypothesis is that the number of ED visits for some infectious diseases is associated with ambient air pollution concentration levels. There are two main arguments which support these associations which gave rise to this hypothesis.

The first argument is that ambient air pollutants may increase the occurrence of bacteria, viruses, or other pathogens in ambient air pollution [10]. This may be due to the presence of specific components in urban particulate matters, chemical reactions and properties (such as $\mathrm{pH}$ values and the presence of active metals) of the air pollutants, air temperature and humidity or other characteristics [11-14].

Secondly, ambient air pollution may act as an immunosuppressive factor that can undermine the normal human body defenses $[6,15,16]$.

The schema or path that is followed to justify this study is therefore as follows: ambient air pollution concentration levels <implies $>$ an increase in pathogen concentration in the ambient air and/or decreases immune defenses <implies $>$ infectious diseases <implies $>$ symptoms and signs of a health condition $<$ implies $>$ an increase of ED visits. In this paper we assumed a hypothesis that air quality is related to the occurrence of infectious disease. We considered whole Chapter of the disease classification which covers ED visits for infectious diseases. The authors believe this work is innovative in the approach to study such health outcomes, use one statistical method (quasi-Poisson regression on the hierarchical clusters), use relatively short lag, $0-14$ days.

\section{Materials and Methods}

Health outcomes considered were the diagnosed emergency department (ED) visits in Toronto, Canada in the period from the 1st of April, 2004 to the 31st of December, 2015 (covering 4,292 days). The health data were drawn from the National Ambulatory Care Reporting System database [17]. The database was queried by using ICD-10 codes A00B99 to identify the health cases for this study. Only primary causes of the ED visits were considered and used to retrieve the related health cases. The cases were summarized and the realized statistical analysis was applied using their daily counts.

The population studied constituted of males and females of all ages visiting EDs in Toronto, in the province of Ontario, Canada. The advantage of considering all age groups while maintaining the capacity to separate the cases by age is that elderly populations tend to have a weaker immune system, while younger individuals have been shown to be susceptible to infections in the respiratory tract when exposed to elevated pollution levels. The NACRS database is a reporting health system which provides diagnosed ED visits. The system covers more than $97 \%$ of the ED visits in the province. The data from the NACRS database were drawn using SAS Guide 7.1 software (SAS Institute, Cary, NC, USA). The retrieved records contain basic patient information and among them are sex, age, date of ED visit, cause of visits, and postal code.

The region of the study is well defined by the Census Division (CD) of Toronto. The considered land area is 630.2 square kilometers. The population density of this area is 4334.4 people per square kilometer. The enumerated population of the CD of Toronto in the year 2016 was 2,731,571 people. ED visits by location within the city of Toronto were classified using the alphanumeric characters. These six characters of available postal codes for each patient's home address were applied to include the patients.

The air pollution data were obtained from the National Air Pollution Surveillance (NAPS), maintained by Environment and Climate Change Canada (NAPS, [18]). Hourly 
data from nine air pollution monitor stations were averaged to estimate the air pollution concentration level for the whole city. Daily values of air pollutants and weather factors were organized as a dataset. This dataset has already been used in other studies related to ambient air pollution concentration levels, temperature, and relative humidity to assess short term mortality risks and excess mortality in 406 locations in 20 countries, including Canada and Toronto [19].

Six urban ambient air pollutants were considered: carbon monoxide (CO), nitrogen dioxide $\left(\mathrm{NO}_{2}\right)$, ground-level ozone $\left(\mathrm{O}_{3}\right.$ - as a daily average, $\mathrm{O}_{3}-8$ hour ozone $\left(\mathrm{O}_{3} \mathrm{H} 8\right)$ - as a maximum eight hour average), fine particulate matter $\left(\mathrm{PM}_{2.5}\right)$, and sulphur dioxide $\left(\mathrm{SO}_{2}\right)$.

In addition to these six air pollutants, the Air Quality Health Index (AQHI), based on three individual ambient air pollutants considered together, $\mathrm{O}_{3}, \mathrm{NO}_{2}$, and $\mathrm{PM}_{2.5}$ was also investigated. The index values incorporate air pollutant concentration levels and the health risk estimations determined by mortality rates in large Canadian cities [20]. The $\mathrm{AQHI}$ values are calculated according to the following formula.

$$
A Q H I=\frac{1000}{10.4} \times\left(e^{0.000537 * 03}+e^{0.000871 * N O 2}+e^{0.000487 * P M 2.5}-3\right) .
$$

In this analysis continuous values obtained by this formula were used. For the Canadian public, these values are rounded and shown as integer numbers on a scale $(1-10,10+)$ to represent the risk related to ambient air quality. In addition, another value of the index, here called the AQHIX, was estimated using $\mathrm{O}_{3}-8$ hour ozone concentration levels and the above formula. This index has stronger representation of ozone (it is an 8-hour maximum rather than a daily average) among its three air pollutant components than in the AQHI calculations. The constructed indexes were applied to realize multipollutant exposures. These are air quality health indexes which in their construction contain three ambient air pollutant concentrations.

The case-crossover technique used in this study controls for all measured and unmeasured time-invariant confounders by design, such as socioeconomic position, smoking or comorbidity [21]. In the fitted models, temperature and relative humidity were incorporated in the form of natural splines. The time-stratified approach was used to group the data by the same day of week in one month [22]. The coefficients (slope, Beta) related to air pollutants and their standard errors were determined. Using these values, relative risks could be easily estimated. The statistical calculations were realized as conditional quasi-Poisson regression models $[23,24]$ The calculations were performed in $\mathrm{R}$ statistical software using the procedure gnm (generalized non-linear models) with the "quasipoisson" option [25]. The statistical models were constructed using hierarchical clusters of the following form based on the calendar structure: (year:month:day of week). This is done to control time and time related changes in the analyzed time-series data (daily counts of ED visits, concentration of air pollutants, weather factors). In total, 2,160 statistical models $\{15$ (lags) x 18 (strata) x 8 (air pollutants) were constructed. The analyses were done realizing the statistical models of the following forms

ModelINF $=$ gnm $($ EDInf $\sim$ APollutant + ns $($ RelativeHumidity,3 $)+$ ns $($ Temperature,3 $)$.

The options data=EDVisitsInf, family=quasipoisson, eliminate=factor(Cluster) were specified. EDInf represent daily counts for the considering strata. The quasi-Poisson is applied to model an overdispersed count variable.

The strata were separated by sex, age group (under 11, between 11 and 60, over 60 years), and season (warm: April-September, cold: October-March). The results from all models are listed in Supplementary Materials. A p-value $<0.05$ was considered statistically significant. These model results were also compared with results using a p-value < 0.001 .

As the results are generated in a $3 \mathrm{D}$ space $(x-y-z)$ determined by air pollutants, their lags, and strata, we constructed a series of maps of the associations. The maps represent a summary of all positive associations for the specified axis. For example, on the plane <air pollution - lags> are shown values for all 18 strata combined. 
In addition to verify the associations the considered health group (Chapter I: "Certain infectious and parasitic diseases" -A00-B99) was split into two collections of health conditions identified by the ICD-10 codes A00-A99 and B00-B99. The analysis was done separately for each subgroup using the same approach as for whole chapter.

\section{Results}

The results are based on ED visits identified using ICD-10 codes A00-B99 ("Certain infectious and parasitic diseases"). In the period of the study, 339,644 such ED visits were identified, and among them 177,619 were for females and 162,025 for males. Table 1 summarizes the statistics on the daily counts of ED visits for infectious diseases by the 18 strata constructed.

Table 1. Descriptive statistics on daily emergency department (ED) visits for infectious diseases (ICD-10: A00-B99). Toronto, Canada, April 2004 - December 2015.

\begin{tabular}{llllllll}
\hline Strata & ED visits & Min & Q1 & Median & Mean & Q3 & Max \\
\hline All & 339,644 & 19 & 45 & 79 & 79.1 & 105 & 264 \\
Female & 177,619 & 4 & 22 & 41 & 41.4 & 56 & 139 \\
Male & 162,025 & 5 & 22 & 37 & 37.8 & 50 & 125 \\
Warm All & 161,578 & 20 & 43 & 77 & 73.6 & 98 & 177 \\
Warm Female & 84,446 & 6 & 22 & 40 & 38.5 & 52 & 96 \\
Warm Male & 77,132 & 5 & 21 & 35 & 35.1 & 46 & 87 \\
Cold All & 178,066 & 19 & 47 & 83 & 85.0 & 116 & 264 \\
Cold Female & 93,173 & 4 & 24 & 43 & 44.5 & 62 & 139 \\
Cold Male & 84,893 & 6 & 24 & 39 & 40.5 & 55 & 125 \\
Age 0-10 All & 95,377 & 1 & 13 & 20 & 22.2 & 29 & 111 \\
Age 0-10 Female & 42,933 & 0 & 6 & 9 & 10.0 & 13 & 53 \\
Age 0-10 Male & 52,444 & 0 & 7 & 11 & 12.2 & 16 & 58 \\
Age 11-60 All & 185,907 & 7 & 24 & 43 & 43.3 & 58 & 130 \\
Age 11-60 Female & 100,324 & 2 & 13 & 23 & 23.4 & 32 & 77 \\
Age 11-60 Male & 85,583 & 1 & 12 & 19 & 19.9 & 27 & 66 \\
Age 60+All & 58,360 & 0 & 7 & 13 & 13.6 & 19 & 45 \\
Age 60+ Female & 34,362 & 0 & 4 & 7 & 8.0 & 12 & 32 \\
Age 60+ Male & 23,998 & 0 & 3 & 5 & 5.6 & 8 & 20 \\
\hline
\end{tabular}

Notes. Column labelled as "ED visits" shows the number of ED visits, Warm: April - September, Cold: October - March, Min - minimum, Max -maximum, Q1-25th percentile, Q375th percentile.

In the warm period (April-September), there were 161,5786 visits and in the cold period (October-March) there were 178,066 ED visits. There were 95,377 visits among young persons (under 11 years), 185,907 among persons between 11 and 60 years of age, and 58,360 visits among older individuals. Table 2 summarizes and presents the statistics on ambient air pollutants, temperature, and relative humidity for the considered period of the study. 
Table 2. Statistics on environmental factors (4,292 days) considered in this study. Toronto, Canada, April 2004 - December 2015.

\begin{tabular}{llllll}
\hline Factors & Units & Q1 & Median & Mean & Q3 \\
\hline $\mathrm{PM}_{2.5}$ & $\mu \mathrm{g} / \mathrm{m}^{3}$ & 4.7 & 7.1 & 8.9 & 11.2 \\
$\mathrm{NO}_{2}$ & $\mathrm{ppb}$ & 11.1 & 15.0 & 16.1 & 19.9 \\
$\mathrm{O}_{3}$ & $\mathrm{ppb}$ & 16.8 & 23.0 & 23.5 & 29.6 \\
$\mathrm{O}_{3} \mathrm{H} 8$ & $\mathrm{ppb}$ & 33.0 & 41.0 & 43.7 & 52.0 \\
$\mathrm{SO}_{2}$ & $\mathrm{ppb}$ & 0.5 & 1.0 & 1.4 & 1.7 \\
$\mathrm{CO}$ & $\mathrm{ppm}$ & 0.2 & 0.2 & 0.3 & 0.3 \\
$\mathrm{AQHI}$ & number & 2.4 & 2.9 & 3.0 & 3.4 \\
AQHIX & number & 3.6 & 4.2 & 4.4 & 5.1 \\
Temperature & ${ }^{\circ} \mathrm{C}$ & 1.7 & 10.0 & 9.5 & 18.4 \\
Relative Humidity & $\%$ & 63.9 & 70.9 & 70.7 & 78.2 \\
\hline
\end{tabular}

Notes. Q1-25th percentile, Q3-75th percentile.

Figure 1,2, and 3 show the numbers of positive statistically significant associations (the coefficient Beta is positive) obtained for the specifications (stratum, air pollutant, lag) given in the corresponding row and column. The cells show the total number of such associations. For example, on Figure 1, for a row identified as "All" and a column identified as "lag 5", the value provided in the cell with such coordinates gives the number of the positive associations for all pollutants combined. In this case there are 4 positive associations. The colors in Figure 1-3 are applied highlight the patterns of the associations. When applying a more stringent $p$-value criterion $(<0.001)$ we observed 27 positive and statistically significant associations. Thus only $10 \%$ of the positive statistically significant associations persisted.

Figure 1 is constructed with the 18 strata in rows and 15 lags (0-14) in columns. Figure 1 illustrates the counts of positive statistically significant associations for the strata and lags. The concentrations lagged by 1,3 , and 5 days have the largest impact; 34,32 , and 35, respectively, of the positive associations among all 279 identified.

\begin{tabular}{lrrrrrrrrrrrrrrrrr|r|} 
& Lag & 0 & 1 & 2 & 3 & 4 & 5 & 6 & 7 & 8 & 9 & 10 & 11 & 12 & 13 & 14 & Total \\
All & 1 & 3 & 3 & 2 & 2 & 4 & 0 & 1 & 1 & 1 & 1 & 0 & 2 & 3 & 0 & 24 \\
Female & 1 & 1 & 1 & 2 & 1 & 5 & 0 & 0 & 3 & 3 & 1 & 0 & 0 & 2 & 0 & 20 \\
Male & 1 & 3 & 3 & 4 & 1 & 1 & 0 & 1 & 1 & 0 & 0 & 0 & 1 & 2 & 0 & 18 \\
Warm All & 1 & 7 & 4 & 4 & 4 & 3 & 1 & 1 & 1 & 1 & 0 & 0 & 0 & 1 & 0 & 28 \\
Warm Female & 1 & 2 & 2 & 3 & 2 & 1 & 1 & 1 & 1 & 2 & 1 & 1 & 0 & 1 & 2 & 21 \\
Warm Male & 0 & 6 & 2 & 2 & 3 & 3 & 3 & 1 & 1 & 0 & 0 & 0 & 1 & 0 & 0 & 22 \\
Cold All & 1 & 1 & 1 & 1 & 0 & 0 & 1 & 1 & 0 & 0 & 0 & 0 & 0 & 0 & 0 & 6 \\
Cold Female & 1 & 1 & 1 & 1 & 0 & 4 & 0 & 0 & 1 & 1 & 2 & 0 & 0 & 0 & 0 & 12 \\
Cold Male & 2 & 1 & 1 & 1 & 0 & 0 & 1 & 0 & 1 & 0 & 0 & 0 & 0 & 0 & 0 & 7 \\
Age 0-10 All & 1 & 1 & 4 & 2 & 2 & 1 & 1 & 0 & 3 & 4 & 1 & 4 & 3 & 4 & 4 & 35 \\
Age 0-10 Female & 1 & 1 & 2 & 3 & 2 & 2 & 0 & 0 & 3 & 4 & 1 & 2 & 0 & 4 & 3 & 28 \\
Age 0-10 Male & 0 & 1 & 1 & 0 & 0 & 1 & 0 & 0 & 0 & 0 & 0 & 1 & 2 & 1 & 1 & 8 \\
Age 11-60 All & 1 & 2 & 1 & 2 & 1 & 1 & 0 & 1 & 1 & 1 & 1 & 0 & 0 & 0 & 0 & 12 \\
Age 11-60 Female & 0 & 1 & 1 & 1 & 0 & 4 & 1 & 0 & 0 & 0 & 1 & 0 & 0 & 0 & 0 & 9 \\
Age 11-60 Male & 1 & 1 & 0 & 2 & 1 & 0 & 0 & 1 & 1 & 1 & 0 & 0 & 0 & 1 & 0 & 9 \\
Age 60+All & 3 & 1 & 0 & 0 & 0 & 1 & 0 & 0 & 0 & 1 & 0 & 0 & 0 & 0 & 0 & 6 \\
Age 60+ Female & 3 & 0 & 0 & 0 & 0 & 0 & 0 & 0 & 1 & 1 & 0 & 0 & 0 & 0 & 0 & 5 \\
Age 60+ Male & 1 & 1 & 0 & 2 & 0 & 4 & 0 & 0 & 0 & 0 & 0 & 1 & 0 & 0 & 0 & 9 \\
& Total & 20 & 34 & 27 & 32 & 19 & 35 & 9 & 8 & 19 & 20 & 9 & 9 & 9 & 19 & 10 & 279 \\
\hline
\end{tabular}

Figure 1. The results for all pollutants combined. Total counts of positive associations: strata (rows), lags (columns). Toronto, Canada. 2004-2015.

Figure 2 is build with 8 air pollutants (rows) and 15 lags (columns). Figure 2 indicates that sulphur dioxide $\left(\mathrm{SO}_{2}\right)$ has the highest number of positive statistically significant associations. In total there are 72 positive associations for $\mathrm{SO}_{2}$. 


\begin{tabular}{lrrrrrrrrrrrrrrrrrr}
\multicolumn{1}{c}{ Lag } & 0 & 1 & 2 & 3 & 4 & 5 & 6 & 7 & 8 & 9 & 10 & 11 & 12 & 13 & 14 & Total \\
$\mathrm{AQHI}$ & 0 & 3 & 2 & 0 & 0 & 4 & 1 & 0 & 2 & 3 & 0 & 2 & 3 & 2 & 2 & 24 \\
$\mathrm{AQHIX}$ & 0 & 2 & 2 & 3 & 2 & 8 & 1 & 0 & 0 & 2 & 0 & 2 & 1 & 4 & 2 & 29 \\
$\mathrm{CO}$ & 2 & 3 & 5 & 13 & 7 & 6 & 0 & 0 & 0 & 0 & 0 & 0 & 0 & 0 & 1 & 37 \\
$\mathrm{NO}_{2}$ & 0 & 2 & 2 & 4 & 3 & 4 & 1 & 0 & 0 & 1 & 0 & 2 & 5 & 8 & 3 & 35 \\
$\mathrm{O}_{3}$ & 13 & 11 & 7 & 0 & 0 & 1 & 2 & 1 & 3 & 5 & 7 & 1 & 0 & 0 & 0 & 51 \\
$\mathrm{O}_{3} \mathrm{H} 8$ & 3 & 5 & 0 & 1 & 0 & 2 & 0 & 0 & 0 & 2 & 1 & 0 & 0 & 0 & 0 & 14 \\
$\mathrm{PM}_{2.5}$ & 0 & 4 & 3 & 1 & 0 & 3 & 1 & 0 & 3 & 2 & 0 & 0 & 0 & 0 & 0 & 17 \\
$\mathrm{SO}_{2}$ & 2 & 4 & 6 & 10 & 7 & 7 & 3 & 7 & 11 & 5 & 1 & 2 & 0 & 5 & 2 & 72 \\
$\quad$ Total & 20 & 34 & 27 & 32 & 19 & 35 & 9 & 8 & 19 & 20 & 9 & 9 & 9 & 19 & 10 & 279
\end{tabular}

Figure 2. The results for all strata combined. Total counts of positive associations: air pollutants (rows), lags (columns). Toronto, Canada. 2004-2015.

Figure 3 shows the results for 18 strata and the 8 air pollutants. The highest value was obtained for the 'Age 0-10 All', with a value of 35 .

\begin{tabular}{|c|c|c|c|c|c|c|c|c|c|}
\hline Air Pollution: & 完 & $\underset{\&}{T}$ & O & ָ̃ & o & 裔 & $\sum_{a}^{N}$ & Ō & Total \\
\hline All & 2 & 2 & 4 & 2 & 4 & 1 & 0 & 9 & 24 \\
\hline Female & 2 & 2 & 3 & 2 & 6 & 1 & 2 & 2 & 20 \\
\hline Male & 1 & 1 & 2 & 3 & 2 & 1 & 2 & 6 & 18 \\
\hline Warm All & 1 & 5 & 5 & 6 & 0 & 1 & 1 & 9 & 28 \\
\hline Warm Female & 1 & 0 & 6 & 5 & 3 & 0 & 0 & 6 & 21 \\
\hline Warm Male & 1 & 5 & 1 & 6 & 0 & 1 & 1 & 7 & 22 \\
\hline Cold All & 0 & 0 & 1 & 0 & 5 & 0 & 0 & 0 & 6 \\
\hline Cold Female & 1 & 1 & 2 & 0 & 6 & 1 & 1 & 0 & 12 \\
\hline Cold Male & 0 & 0 & 1 & 0 & 4 & 1 & 0 & 1 & 7 \\
\hline Age 0-10 All & 8 & 5 & 3 & 5 & 2 & 0 & 3 & 9 & 35 \\
\hline Age 0-10 Female & 5 & 5 & 2 & 2 & 3 & 1 & 4 & 6 & 28 \\
\hline Age 0-10 Male & 1 & 0 & 0 & 2 & 2 & 0 & 0 & 3 & 8 \\
\hline Age 11-60 All & 0 & 0 & 3 & 0 & 4 & 1 & 0 & 4 & 12 \\
\hline Age 11-60 Female & 1 & 0 & 2 & 1 & 3 & 0 & 2 & 0 & 9 \\
\hline Age 11-60 Male & 0 & 0 & 1 & 0 & 1 & 1 & 0 & 6 & 9 \\
\hline Age $60+$ All & 0 & 1 & 0 & 0 & 2 & 1 & 0 & 2 & 6 \\
\hline Age 60+ Female & 0 & 0 & 0 & 0 & 3 & 1 & 0 & 1 & 5 \\
\hline Age 60+ Male & 0 & 2 & 1 & 1 & 1 & 2 & 1 & 1 & 9 \\
\hline Total & 24 & 29 & 37 & 35 & 51 & 14 & 17 & 72 & 279 \\
\hline
\end{tabular}

Figure 3. The results for all lags combined. Total counts of positive associations: strata (rows), air pollutants (columns). Toronto, Canada. 2004-2015.

Figure 4 is a sort of a map to the table with numerical estimations (slope and its standard error). For each air pollutant Figure 4 shows the associations with the following values: 0 (none), statistically significant; -1 (negative), and 1 (positive). Colours are used to distinguish the relations (white, green, and red, respectively). The columns represent lags 
from 0 to 14. The corresponding numerical values are available as Supplementary Materials at GitHub - szyszkowiczm/INFECTIOUSToronto in the file INFECTIONToronto.csv. The file presents 2,160 coefficients (BETA) and their standard errors (SEBeta). The used lags are indicated in the rows labelled as "All LAG $\mathrm{n}$ ", where $\mathrm{n}$ is the number of days.

AQHI

\begin{tabular}{|c|c|c|c|c|c|c|c|c|c|c|c|c|c|c|c|c|c|c|c|c|c|c|c|c|c|c|c|}
\hline & 0 & 0 & 0 & 0 & & 1 & 00 & 0 & 0 & 0 & 0 & & 0 & 0 & 0 & 0 & 1 & 1 & 1 & 1 & 0 & 0 & 0 & 0 & & & \\
\hline Female & 0 & 0 & 0 & 0 & 0 & 1 & $0 \quad 0$ & 0 & 1 & 0 & 0 & 0 & 0 & t & 0 & 0 & 0 & 1 & 1 & 1 & 0 & 0 & 0 & 0 & 0 & 00 & \\
\hline ale & 0 & 0 & 1 & 0 & 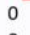 & 0 & 00 & 0 & 0 & 0 & 0 & 0 & 0 & 0 & 0 & 0 & 1 & 1 & 0 & 0 & 0 & 0 & 0 & 0 & 0 & 00 & 0 \\
\hline arm All & 0 & 1 & 0 & 0 & 0 & 0 & 0 & 0 & 0 & 0 & 0 & 0 & 0 & 0 & 1 & 1 & 1 & 1 & 1 & 0 & 0 & 0 & 0 & 0 & 0 & 00 & 0 \\
\hline arm Female & 0 & 1 & 0 & 0 & 0 & 0 & 0 & 0 & 0 & 0 & 0 & 0 & 0 & 0 & 1 & 1 & 1 & 1 & 1 & 0 & 0 & 0 & 0 & 0 & 0 & 00 & 0 \\
\hline arm Male & 0 & 1 & 0 & 0 & 0 & 0 & 0 & 0 & 0 & 0 & 0 & 0 & 0 & 0 & 0 & 1 & 0 & 0 & 0 & 0 & 0 & 0 & 0 & 0 & 0 & 00 & 0 \\
\hline d All & -1 & 0 & 0 & 0 & 0 & 0 & 0 & 0 & 0 & 0 & 0 & 0 & 0 & 0 & 0 & 0 & 0 & 1 & 0 & 0 & 0 & 0 & 0 & 0 & 0 & 00 & 0 \\
\hline$m a$ & 0 & 0 & 0 & 0 & 0 & 1 & 0 & 0 & 0 & 0 & 0 & 0 & 0 & 0 & 0 & 0 & 0 & 1 & 0 & 0 & 00 & 0 & 0 & 0 & 0 & 00 & 00 \\
\hline Id Male & 0 & 0 & 0 & 0 & 0 & 0 & 0 & 0 & 0 & 0 & 0 & 0 & 0 & 0 & 0 & 0 & 1 & 1 & 1 & 0 & 0 & 0 & 0 & 0 & 0 & 00 & 0 \\
\hline $0-10 \mathrm{~A}$ & 0 & $\begin{array}{l}0 \\
0\end{array}$ & 1 & 0 & 0 & 0 & 1 & 1 & 1 & 0 & 1 & 1 & 1 & & 0 & 0 & 0 & 1 & 1 & 0 & 0 & 0 & 0 & 0 & 0 & 00 & 0 \\
\hline Age 0-10 Male & 0 & 0 & 0 & 0 & 0 & 0 & 0 & 0 & 1 & 0 & 1 & 0 & $\begin{array}{l}1 \\
0\end{array}$ & & 0 & 0 & 0 & 0 & 0 & 0 & 0 & 0 & 0 & 0 & 0 & 00 & 0 \\
\hline e 11-6c & 0 & c & 0 & 0 & 0 & 0 & 0 & 0 & 0 & 0 & 0 & 0 & 0 & & 0 & 0 & 0 & 1 & 1 & 1 & 0 & 0 & 0 & -1 & & 00 & 0 \\
\hline $11-60$ & 0 & 0 & 0 & 0 & 0 & 1 & 0 & 0 & 0 & 0 & 0 & 0 & 0 & 0 & 0 & 0 & 0 & 1 & 0 & 1 & 00 & 0 & 0 & 0 & & 00 & 0 \\
\hline $1-60>->$ & 0 & 0 & 0 & 0 & 0 & 0 & ( & 0 & 0 & 0 & 0 & 0 & 0 & 0 & 0 & 0 & 0 & 1 & 0 & 0 & 00 & 0 & 0 & -1 & & 00 & 0 \\
\hline e 60+All & 0 & 0 & 0 & 0 & 0 & 0 & 0 & 0 & 0 & 0 & 0 & 0 & 0 & 0 & 0 & 0 & 0 & 0 & 0 & 0 & 0 & 0 & 0 & 0 & 0 & 00 & \\
\hline e $60+\mathrm{Fem}$ & 0 & 0 & 0 & 0 & 0 & 0 & 0 & 0 & 0 & 0 & 0 & 0 & 0 & 0 & 0 & 0 & 0 & 0 & 0 & 0 & $0-1$ & $\begin{array}{ll}-1 & 0\end{array}$ & 0 & 0 & 0 & 00 & 0 \\
\hline ge $60+$ Male & 0 & 0 & 0 & 0 & 0 & 0 & 0 & 0 & & 0 & 0 & 0 & 0 & 0 & 0 & 0 & 0 & 0 & & 1 & & 0 & 0 & 0 & & 00 & 0 \\
\hline
\end{tabular}

\section{AQHIX}

$\mathrm{NO} 2$

\section{$\begin{array}{lllllllllllllll}0 & 0 & 0 & 0 & 0 & 1 & 0 & 0 & 0 & 0 & 0 & 0 & 0 & 1 & 0\end{array}$}

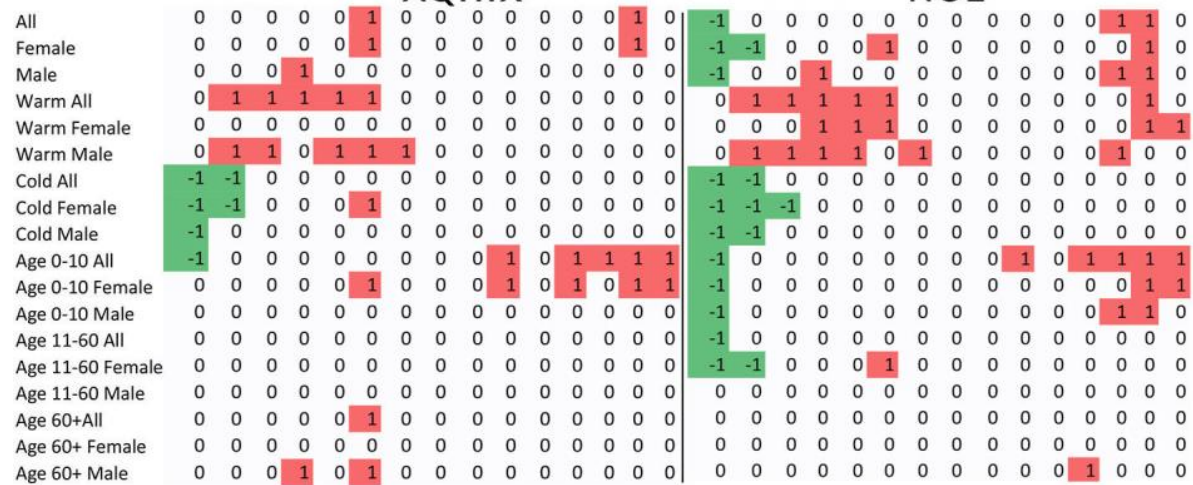

Age 60+ Male

\section{3}

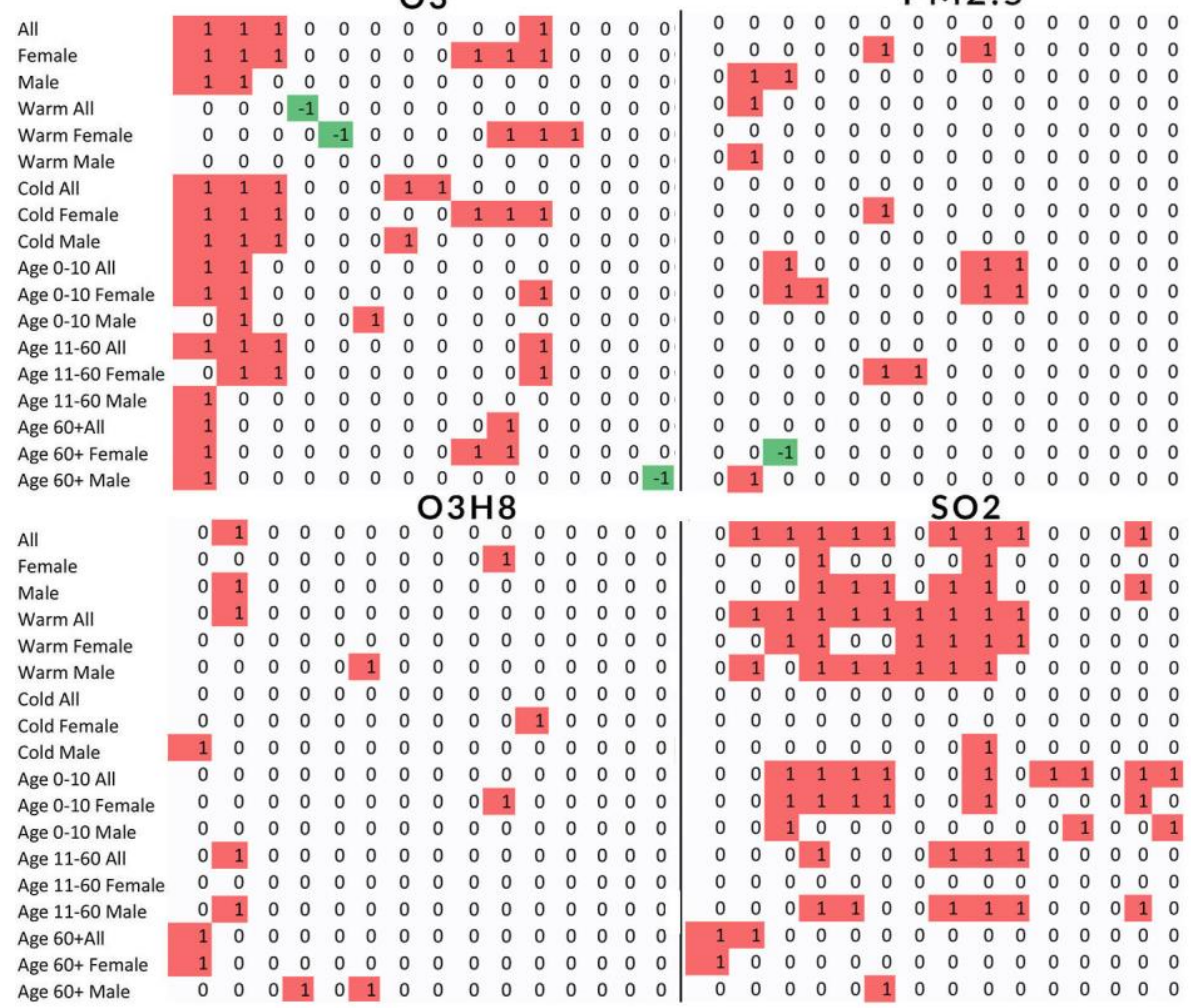

Figure 4. The results from 2,160 models shown as a map to numerical results; strata (rows), lags (columns). 0/white - no associations, and statistically significant: -1/green - negative, 1/red - positive. The results are grouped by air pollutants. Toronto, Canada. 2004-2015. 
In this work the values of relative risks are not reported explicitly as so many models were tested. The numerical results for all models are provided in Supplementary Materials (file INFECTIONToronto.csv). Using this information, values given for air pollutants in Table 2, coefficients (BETA) and their standard errors (SEBeta) from the file INFECTIONToronto.csv, it is easy to calculate the relative risk (RR). For example, for All patients, for the same day exposure to ozone (lag 0), an increase in a one interquartile range (IQR=12.8 ppb, see Table 2), gives $\mathrm{RR}=1.028$ with $95 \%$ confidence interval (95\%CI: 1.014, 1.042), where the estimated parameters are BETA $=0.002146$ and SEBeta $=0.000537$. In the file InfectionRRiskTORONTO.csv all values of RR and theirs 95\% confidence intervals are presented (Supplementary Materials).

As sulphur dioxide shows the highest number of positive associations among the considered air pollutants the relative risks for its concentration increase are reported here. The following values were estimated for All: RR=1.005 (95\% CI: 0.998, 1.013), 1.008 (1.001, 1.016), 1.009 (1.001, 1.016), 1.011 (1.004, 1.019), 1.007 (0.987, 1.028), and 1.009 (1.002, 1.016) for lags from 0 to 5 , respectively.

As we observed many positive statically significant associations between the number of ED visits and carbon monoxide, we created Figure 5. The latter is a forest plot and represents the estimated RR and their 95\%CIs as bars and shows their numerical values. The results are reported for an increase of $1.2 \mathrm{ppm}$ in $\mathrm{CO}$ concentration level.

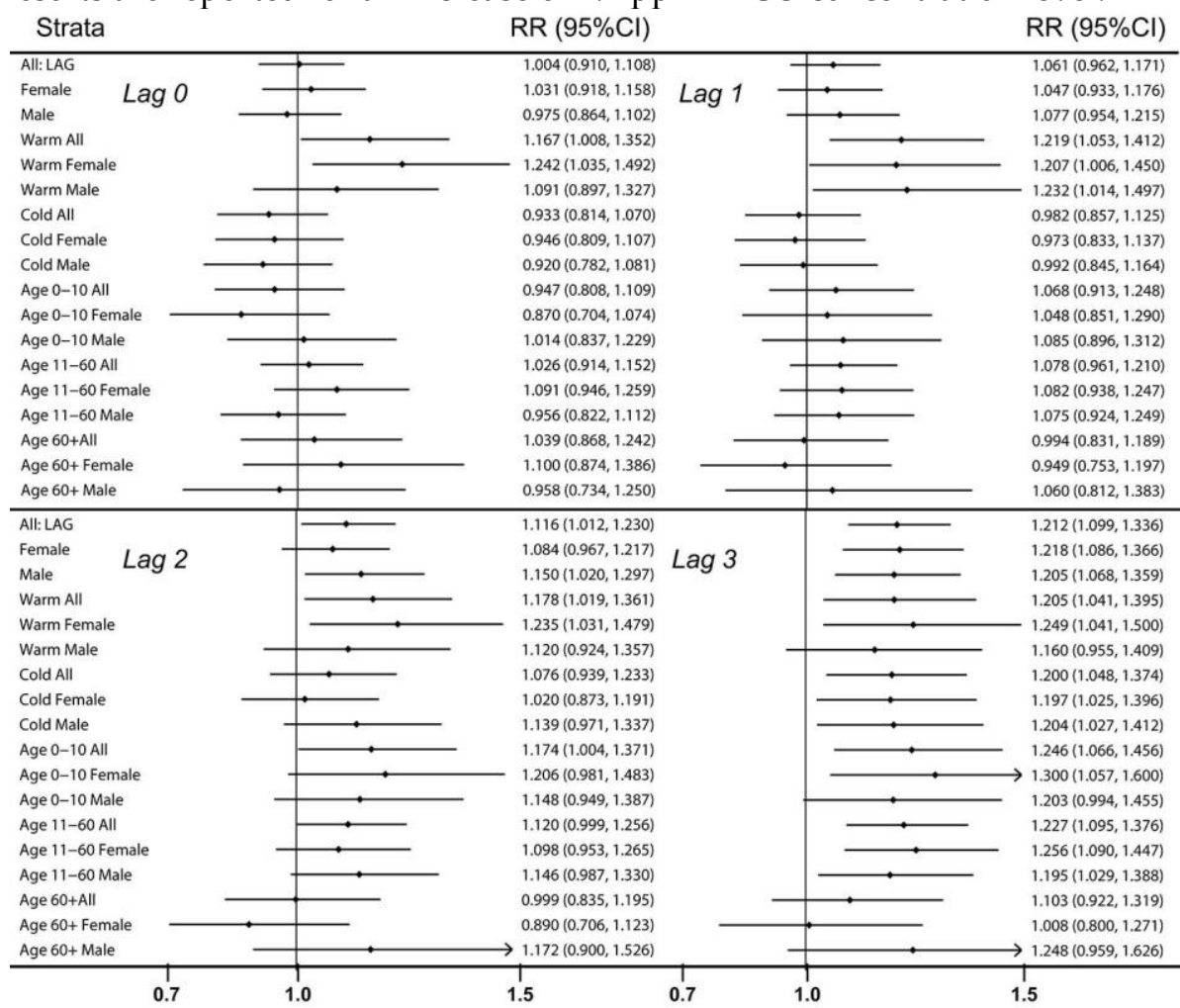

Figure 5. A forest plot of RR and their $95 \%$ CIs for carbon monoxide for lag $0,1,2$, and 3 days. Toronto, Canada. 2004-2015.

Table 3 presents the most frequent ED visits identified by three characters of the ICD10 codes. The ED visits diagnosed with ICD-10 code A09 ("Other gastroenteritis and colitis of infectious and unspecified origin") have the highest count with 106,784 cases, which is $31.4 \%$ of all the considered ED visits. The second position belongs to the ED visits described as "Viral infection of unspecified site" with the code B34. There are 97,740 such visits, or $28.8 \%$ of all visits. 
Table 3. The most frequent ED visits diagnosed with the ICD-10 codes in the range A00-B99. Toronto, Canada, 2004 - 2015.

\begin{tabular}{llll}
\hline Description & ICD10 & Counts & \% \\
\hline Other gastroenteritis and colitis of infectious and uns. origin & A09 & 106,784 & 31.4 \\
Viral infection of unspecified site & B34 & 97,740 & 28.8 \\
Viral and other specified intestinal infections & A08 & 29,726 & 8.8 \\
Other sepsis & A41 & 17,292 & 5.1 \\
Zoster [herpes zoster] & B02 & 16,029 & 4.7 \\
Candidiasis & B37 & 6,908 & 2.0 \\
Herpesviral [herpes simplex] infections & B00 & 6,366 & 1.9 \\
Uns. viral infection characterized by skin and muc. m. lesions & B09 & 4,899 & 1.4 \\
Varicella [chickenpox] & B01 & 4,282 & 1.3 \\
Other bacterial intestinal infections & A04 & 4,208 & 1.2 \\
Other predominantly sexually transmitted diseases, nec & A63 & 4,205 & 1.2 \\
Other viral inf. characterized by skin and muc. m. lesions, nec & B08 & 4,096 & 1.2 \\
Dermatophytosis & B35 & 3,674 & 1.1 \\
Viral conjunctivitis & B30 & 3,551 & 1.1 \\
\hline
\end{tabular}

The map of the city of Toronto with population density and the location of monitor stations is given in Supplementary Materials. Also the figures with the frequencies of air pollutants and weather factors are presented.

In the additional analysis there were 176,108 ED visits identified by the codes A00A99 and 163,536 ED visits identified by the codes B00-B99 for all patients. The following relative risks and $95 \% \mathrm{CIs}$ were estimated for sulphur dioxide (IQR=1.2 ppb) $1.004(0.986$, 1.023), 1.008 (0.990, 1.026), 1.009 (0.990, 1.028), 1.004 (0.985, 1.023), 1.014 (0.995, 1.032), 1.004 $(0.986,1.023)$, and $0.999(0.980,1.018)$, for lags $0-6$. The analogous results for the group B00-B99 were $0.990(0.975,1.005), 1.006$ (0.990, 1.021), 1.013 (0.998, 1.029), 1.017 (1.001, $1.032), 1.023(1.008,1.038), 1.018(1.003,1.034)$, and $1.018(1.002,1.034)$. The results for all 2,160 models are given in Supplementary Materials. Also the corresponding figures to Figs. 1-3 are included.

\section{Discussion}

The objective of the presented paper was to verify the associations between the number of ED visits for infectious disease and urban ambient air pollution. Among the 2,160 constructed models, 279 of them show positive statistically significant associations. It is almost $13 \%$ of the all tested correlations. In such a large number of tests, the main idea is to identify a pattern among the relations. It is expected, if the associations do really exist, to see some confirmations for the used strata, lags or air pollutants. Here, air pollution concentrations lagged by 1,3 , and 5 days have the highest number of significant associations. When sorting by air pollutants, sulphur dioxide is identified as the air pollutant with the highest number of positive, statistically significant associations. We acknowledge that causality can not be proven in such a study as it is only a statistical assessment. To the best of the author's knowledge the relationship between infectious disease risk outside of the respiratory system associated with exposure to ambient air pollution has not been thoroughly explored. It should be noted that these infectious diseases were not classified in the "Diseases of the respiratory system" Chapter. There are many papers published on air pollution causing inflammation and oxidative stress in experimental and epidemiological panel studies [26,27]. Their findings support the hypothesis considered in this study. There are many related publications on respiratory infections [28,29], however this study does not reaffirm what Horne and Croft et al. have established in studies related to respiratory issues, as we look at only the diseases classified by ICD-10 codes A00-B99. 
Not all ED visits for infectious diseases are associated with ambient air pollution concentration levels. A large portion of them could have no relations to air quality and not be triggered by concentration levels. In such situations statistical models should estimate the coefficients (slope, Beta) as zero, i.e. neutral associations. In general, let A be a set of diseases related to air pollution, $B$ as unrelated diseases, and $C$ as negatively related (protective). Such scenarios are common. It is expected that groups $A, A+B$ will give positive associations, whereas $A+C$ will result in neutral outcomes, and $C, B+C$ will result in negative associations. In the obtained results nitrogen dioxide (mainly for lag 0 ) shows negative statistically significant associations. In the considered health outcomes, as it is a large spectrum of diseases ("Certain infectious and parasitic diseases"), the majority of them most likely belong to group B - no associations with urban air pollution. In this work 2,160 models were fitted. A large number of models increases the risk of false positive associations. With a P-value $<0.05,279$ associations were classified as statistically significant and 27 with P-value $<0.001$. Using a similar approach to classify negative associations; there were 31 and 7 , respectively.

The hypotheses of this paper are supported for some combinations of air pollutants and lags. It is interesting that sulphur dioxide shows the most frequent associations. It could be a risk factor by itself or a marker of the presence of other urban ambient air components. A similar analysis was done in Edmonton, Canada for the period April 1991 to March 2002 (3,652 days) using ICD-9 codes (00-139, Chapter: "Infectious and Parasitic Diseases") [30]. In the mentioned paper, positive statistically significant associations for 18 ICD-9 Chapters are presented, for six air pollutants lagged from 0 to 7 days. The results are only shown for All ED visits in the considered Chapter (see Figure 2 in the related publication) [30]. The results in Edmonton also indicate that there is an association of ED visits for infectious disease with ambient air pollution concentration levels, mainly for lags 3,4 , and 5 .

The highest count of sub-chapter ED visit causes was related to infectious colitis, gastroenteritis and enteritis (sub-chapter A09). There have been many studies showing the impact of air pollution on gastrointestinal health, showing that gut flora, increased intestinal permeability and injury to the intestinal lining can predispose the intestine to infection by external pathogens. Air pollutants, particularly PM2.5, contribute to the alteration of normal gut function by the aforementioned pathways [31,32] and can cause increased infection risk [33]. This was not reflected in the results of our study, as PM2.5 had one of the lower association counts at 17 in contrast to the highest amount of associations with $\mathrm{SO}_{2}$ at 72 . To the author's knowledge, there are limited studies examining the impact of $\mathrm{SO}_{2}$ on gut infections and infectious colitis or gastroenteritis, and $\mathrm{SO}_{2}$ has even been shown to reduce the risk of ulcerative colitis [34]. However, there are studies showing a positive association between enteritis and elevated sulfur dioxide concentrations. Xu et al. [35] showed that $\mathrm{SO}_{2}$ and $\mathrm{NO}_{2}$ concentrations have a statistically significant $(p<0.05)$ association with elevated ED visits for enteritis at short lags in Xi'an, China, which is reflected in the results obtained here. A study performed in Edmonton on the frequency of the ED visits due to colitis among young patients (age $<15$ years, $N=11,110$ ) indicated the positive statistically significant associations with particulate matter, carbon monoxide, nitrogen dioxide, and sulphur dioxide. Their findings indicate that odds ratios were elevated for lags 3 and 4 [36]. The biological link between $\mathrm{SO}_{2}$ and gastrointestinal disease has been explored by Z. Meng in multiple studies [37, 38, 39]. $\mathrm{SO}_{2}$ was shown to have a detrimental effect on multiple organ systems. $\mathrm{SO}_{2}$ oxidation can produce oxygen-centered free radicals such as $\mathrm{SO}_{3}^{-}, \mathrm{SO}_{4}^{-}$, and $\mathrm{SO}_{5}^{-}$all of which can interact with DNA, proteins and lipids. These free radicals have been shown to cause chromosomal aberrations, sister chromatid exchanges and micronuclei, through reaction with molecular oxygen forming a highly reactive oxygen species: a peroxyl radical, which can damage nucleotides. These sulfide radicals can also cause lipid peroxidation, known to have a detrimental effect on the structure and function of cell membranes. Given that the findings in these studies were apparent throughout the body, it is possible that the deleterious effect of $\mathrm{SO}_{2}$ exposure may have 
an impact on infections outside the respiratory system. In another work, ambient air pollution was identified as a factor contributing to intestinal disease [40].

The addition calculations for two groups A00-A99 and B00-B99 show the positive statistically significant associations with sulfur dioxide. It is interesting that the similar calculations for ED visits classified under ICD-10 codes J09 - J12 (influenza and viral pneumonia) result with the following values $1.037(1.002,1.074), 1.035(1.000,1.072), 1.053$ $(1.017,1.091), 1.023(0.988,1.060$ for all, lags $0-3$ days, respectively. The associations are also positive statistically significant for lags from 6 to 12. It implies that $\mathrm{SO}_{2}$ has correlations with viral infections.

A very similar approach to study short-term air health effects was used by the authors in China [41]. In a similar way, they also analyzed the health conditions grouped under the chapters of ICD-10 classification. For all infectious diseases (A00-B99) they obtained positive associations for fine particulate matter and ambient ozone [41].

There are some limitations in this study, most quite typical in environmental epidemiology. Two kinds of data were used; health data - here ED visits, and environmental data - air pollutant concentrations and weather factor measurements. The origin of the collected health data may present another risk associated with results inaccuracy. As emergency department data were not collected by the researchers but sourced through the NARCS, the results described here are relying on the quality and accuracy of administratively ascertained data. A result of ED visits falsely attributed to infectious causes contributes to a decrease in the sensitivity of this study. To mitigate these risks, the CIHI (managing body of the NARCS) attempts to ensure that any information it provides goes through the process of capture to dissemination by way of a high-level data management process (General Statistical Business Process Model). Ultimately, the institute can only assure proper data processing, not accurate data collection, building of the health database, data preparation or similar actions related to data processing by hospital staff or other health care practitioners.

This study assumes that all members of the target population have the same level of exposure. Daily air pollution concentration levels were estimated using the fixed monitors in Toronto. Such measurements do not fully represent changes in air pollution concentration exposure among persons living in a large city like Toronto. In this work 2,160 models were fitted, which increases the risk of false positive associations. The adequacy of the statistical models and impact of measurement errors of environmental factors may also influence the results. Numerous hypotheses were tested, but groupings of significant associations for some exposures (strata and lags) and health outcomes were observed. Further studies should focus on identifying subgroups within the ICD-10: B00-B99 (or ICD-9: 00-139) classifications and verify their relation to urban ambient air pollution concentration levels. The results suggest that ambient air pollution may be related to the number of COVID-19 cases [42].

\section{Conclusions}

The exposure from previous days, mainly 5 days before ED visits, has the largest frequency of the statistically positive associations. The presence of sulphur dioxide in the ambient air shows the largest number of the positive associations, and ground level ozone shows the second largest number of such associations. While the AQHI is a standard air quality indicator used in Canada and its definition may not be modified, results obtained in this and other similar studies considering the impact of $\mathrm{SO}_{2}$ suggest that it may be advisable to report ambient $\mathrm{SO}_{2}$ levels as a supplementary air quality measure when addressing the public. In conclusion, based on the results seen in this study, urban ambient air pollution may be related to an increased number of ED visits for certain infectious diseases, however it is important to acknowledge that the associations do not determine causality. 
Supplementary Materials: The numerical estimations are available online at GitHub - szyszkowi$\mathrm{czm} /$ INFECTIOUSToronto in the file INFECTIONToronto.csv. The file contains the coefficients for air pollutants (Beta) and their standard errors (SEBeta) for all constructed models. It allows to calculate relative risks or perform a meta- analysis. These values are given in the file InfectionRRiskTORONTO.csv. The corresponding histograms: HistAQHI-AQHIX-CO-NO2.jpg, HistO3O3H8-PM25-SO2.jpg, HistTempRHum.jpg, and the map of Toronto: TorontoMapStation.jpg. Additional results were included for two considered subgroups identified by the ICD-10 codes A00-A99 and B00-B99; relative risks and 95\% confidence intervals in two files ResA00-A99TorontoRRisk.csv, ResB00-B99TorontoRRisk.csv. The patterns of the positive significant associations are illustrated on the figures A00-A99andB00-B99SumByAirPollutant.jpg, A00-A99andB00-B99SumByLAGS.jpg, and A00-A99andB00-B99SumBySTRATA.jpg.

Author Contributions: Conceptualization, M.S.; methodology, M.S. and N.dA.; software, M.S.; validation, M.S. and N.dA.; formal analysis, M.S. and N.dA.; investigation, M.S.; resources, M.S.; data curation, M.S.; writing - original draft preparation, M.S.; writing-review and editing, N.dA.; visualization, N.dA.; supervision, M.S.; project administration, M.S.; funding acquisition, M.S. All authors have read and agreed to the published version of the manuscript.

Funding: This research received no external funding.

Institutional Review Board Statement: The Health Canada Research Ethic Board determined that the study is IRB exempt, given that patient data were pre-existing and de-identified.

Informed Consent Statement: Not applicable.

Data Availability Statement: Health data: NACRS (2020), The National Ambulatory Care Reporting System, CIHI, Canada; https://www.cihi.ca/en/national-ambulatory-care-reporting-systemmetadata. Environmental data: NAPS (2020) Web site: http://maps-cartes.ec.gc.ca/rnspanaps/data.aspx, Canada.

Acknowledgments: The authors acknowledge Environment Canada for providing the air pollution data from the National Air Pollution Surveillance (NAPS) network.

Parts of this material are based on data and information compiled and provided by the Canadian Institute for Health Information (CIHI). However, the analyses, conclusions, opinions and statements expressed herein are not necessarily those of CIHI.

Conflicts of Interest: The authors declare no conflict of interest. The funders had no role in the design of the study; in the collection, analyses, or interpretation of data; in the writing of the manuscript, or in the decision to publish the results. 


\section{References}

1. Burbank, A.J.; Peden, D.B. Assessing the impact of air pollution on childhood asthma morbidity: how, when, and what to do. Curr Opin Allergy Clin Immunol 2018, 18(2):124-131. doi:10.1097/ACI.0000000000000422.

2. Burnett, R.; Chen, H.; Szyszkowicz, M.; et al. Global estimates of mortality associated with long-term exposure to outdoor fine particulate matter. Proc Natl Acad Sci USA. 2018,115(38):9592-9597. doi: 10.1073/pnas.

3. Szyszkowicz, M.; Thomson, E.M.; Colman, I.; Rowe, B.H. Ambient air pollution exposure and emergency department visits for substance abuse. PLoS One 2018,13(6):e0199826. doi: 10.1371/journal.pone.0199826.

4. Chau, T.; Wang, K. An association between air pollution and daily most frequently visits of eighteen outpatient diseases in an industrial city. Sci Rep 2020, 10, 2321. https://doi.org/10.1038/s41598-020-587210 .

5. Szyszkowicz, M. The Air Quality Health Index and all emergency department visits. Environ Sci Pollut Res Int 2019, 26(24):24357-24361. doi: 10.1007/s11356-019-05741-7.

6. Schraufnagel, D.E.; Balmes, J.R.; Cowl, C.T.; et al. Air Pollution and Noncommunicable Diseases: A Review by the Forum of International Respiratory Societies' Environmental Committee, Part 1: The Damaging Effects of Air Pollution. Chest 2019,155(2):409-416. doi: 10.1016/j.chest.2018.10.042.

7. Thomson, E.M. Air Pollution, Stress, and Allostatic Load: Linking Systemic and Central Nervous System Impacts. Journal of Alzheimer's Disease 2019, 69(3):597-614. doi: 10.3233/JAD-190015.

8. Szyszkowicz, M.; de Angelis, N. Ambient air pollution and emergency department visits in Toronto, Canada. Environ Sci Pollut Res Int 2021, doi: 10.1007/s11356-021-12519-3.

9. ;Szyszkowicz, M.; Schoen, S.; de Angelis, N. Air Pollution and Emergency Department Visits for Disease of the Genitourinary System. Environmental Health Insights 2021, https://doi.org/10.1177/11786302211025360.

10. Frontera, A.; Martin, C.; Vlachos, K.; Sgubind, G. Regional air pollution persistence links to COVID-19 infection zoning. J Infect 2020, doi: 10.1016/j.jinf.2020.03.045.

11. Ge, R.; Sun, X.; He, Q. (2009). Iron acquisition by Streptococcus species: An updated review. Front. Biol. China 2009, 4, 392. https://doi.org/10.1007/s11515-009-0035-4.

12. Fernstrom, A.; Goldblatt, M. Aerobiology and its role in the transmission of infectious diseases. J Pathog 2013, 2013:493960. doi:10.1155/2013/493960.

13. Weinberg, E.D. Iron and infection. Microbiol Rev 1978, 42(1): 45-66. doi: 10.1128/mr.42.1.45-66.1978.

14. Wooldridge, K.G.; Williams, P.H. Iron uptake mechanisms of pathogenic bacteria. FEMS Microbiol Rev 1993, 12(4):325-48. doi: 10.1111/j.1574-6976.1993.tb00026.x.

15. Ciencewicki, J.; Jaspers, I. Air pollution and respiratory viral infection. Inhal Toxicol 2007, 19(14):1135-46. https://doi.org/10.1080/08958370701665434.

16. Glencross, D.A.; Ho, T.R.; Camiña, N.; Hawrylowicz, C.M.; Pfeffer, P.E. Air pollution and its effects on the immune system. Free Radic Biol Med 2020, pii:S0891-5849(19)31521-7. doi: 10.1016/j.freeradbiomed.2020.01.179.

17. NACRS (2021), The National Ambulatory Care Reporting System, CIHI, Canada; https://www.cihi.ca/en/national-ambulatory-care-reporting-system-metadata.

18. NAPS (2021) Web site: http://maps-cartes.ec.gc.ca/rnspa-naps/data.aspx, Canada.

19. Vicedo-Cabrera, A.M.; Sera, F.; Liu, C.; et al. Short term association between ozone and mortality: global two stage time series study in 406 locations in 20 countries. BMJ 2020, 368, https://doi.org/10.1136/bmj.m108.

20. Stieb, D.M.; Burnett, R.T.; Smith-Doiron, M.; Brion, O.; Shin, H.H.; Economou, V. A new multipollutant, no-threshold air quality health index based on short-term associations observed in daily time-series analyses. J Air Waste Manag Assoc 2008, 58(3):435-450. doi:10.3155/1047-3289.58.3.435.

21. Maclure, M. The case-crossover design: a method for studying transient effects on the risk of acute events. Am J Epidemiol 1991, 133:144-153.

22. Janes, H.; Sheppard, L.; Lumley, T. Case-crossover analyses of air pollution exposure data: referent selection strategies and their implications for bias. Epidemiology 2005, 16:717-726.

23. Szyszkowicz, M. Use of generalized linear mixed models to examine the association between air pollution and health outcomes. Int J Occup Med Environ Health 2006, 19:224-227. DOI: 10.2478/v10001-0060032-7.

24. Armstrong, B.G.; Gasparrini, A.; Tobias, A. Conditional Poisson models: a flexible alternative to conditional logistic case cross-over analysis. BMC Med Res Methodol 2014, 14:122. doi: 10.1186/1471-2288-14122.

25. R Core Team. R: A language and environment for statistical computing. R Foundation for Statistical Computing, Vienna, Austria. 2018, URL https://www.R-project.org/. 
26. Liu, L.; Kauri, L.M.; Mahmud, M.; et al. Exposure to air pollution near a steel plant and effects on cardiovascular physiology: a randomized crossover study. Int J Hyg Environ Health 2014, 217(2-3):279-286. doi:10.1016/j.ijheh.2013.06.007.

27. Liu, L.; Urch, B.; Szyszkowicz, M.; et al. Influence of exposure to coarse, fine and ultrafine urban particulate matter and their biological constituents on neural biomarkers in a randomized controlled crossover study. Environ Int 2017, 101:89-95. doi:10.1016/j.envint.2017.01.010.

28. Horne, B.D.; Joy, E.A.; Hofmann, M.G.; et al. Short-Term Elevation of Fine Particulate Matter Air Pollution and Acute Lower Respiratory Infection. Am J Respir Crit Care Med 2018, 198(6):759-766. doi: 10.1164/rccm.201709-1883OC.

29. Croft, D.P.; Zhang, W.; Lin, S.; et al. Associations between Source-Specific Particulate Matter and Respiratory Infections in New York State Adults. Environ Sci Technol 2020, 54(2):975-984. doi: 10.1021/acs.est.9b04295.

30. Szyszkowicz, M.; Rowe, B.H. Respiratory health conditions and ambient ozone: a case-crossover study. Insights Chest Dis 2016, 1:9. http://insightsinchestdiseases.imedpub.com/respiratory-health-conditionsand-ambi.

31. Mutlu, E.A.; Engen, P.A.; Soberanes, S.; et al. Particulate matter air pollution causes oxidant-mediated increase in gut permeability in mice. Part Fibre Toxicol 2011, 8:1-3.

32. van Voorhis, M.; Knopp, S.; Julliard, W.; et al. Exposure to atmospheric particulate matter enhances The polarization through the aryl hydrocarbon receptor. PloS One 2013, 8:e82545.

33. Round, J.L.; Mazmanian, S.K. The gut microbiota shapes intestinal immune responses during health and disease. Nature reviews immunology 2009, 9(5):313-23.

34. Roediger, W.E.; Duncan, A.; Kapaniris, O.; et al. Reducing sulfur compounds of the colon impair colonocyte nutrition: implications for ulcerative colitis. Gastroenterology 1993,104:802-9.

35. Xu, C.; Kan, H.D.; Fan, Y.N.; et al. Acute effects of air pollution on enteritis admissions in Xi'an, China. J Toxicol Environ Health Sci 2016, 79(2):1183-9.

36. Szyszkowicz, M. An approach to represent a combined exposure to air pollution. Int J Occup Med Environ Health 2015;28(5):823-30. doi: 10.13075/ijomeh.1896.00380.

37. Meng, Z. ; Qin, G. ; Zhang, B. ; et al. Oxidative damage of sulfur dioxide inhalation on lungs and hearts of mice. Environmental research 2003, 93:285-292.

38. Meng, Z. Oxidative damage of sulfur dioxide on various organs of mice: sulfur dioxide is a systemic oxidative damage agent. Inhalation toxicology 2003, 15:181-95.

39. Meng, Z.; Qin, G.; Zhang, B.; Bai, J. DNA damaging effects of sulfur dioxide derivatives in cells from various organs of mice. Mutagenesis 2004, 19:465-8.

40. Beamish, L.A.; Osornio-Vargas, A.R.; Wine, E. Air pollution: An environmental factor contributing to intestinal disease. J Crohns Colitis 2011;5(4):279-86. doi: 10.1016/j.crohns.2011.02.017.

41. Gu, J.; Shi, Y.; Zhu, Y.; Chen, N.; Wang, H.; Zhang, Z.; Chen, T. Ambient air pollution and cause-specific risk of hospital admission in China: A nationwide time-series study. PLoS Med 2020, 6;17(8):e1003188. doi: 10.1371/journal.pmed.1003188.

42. Szyszkowicz, M. Modelling the Cumulative Number of COVID-19 Cases. Adv Environ Eng Res 2021, 2(2):10; doi:10.21926/aeer.2102014. 